\title{
Research on the Innovative Design of SAPAD-Based Recreational Products for the Elderly
}

\author{
Liu Xinyi ${ }^{\mathrm{a}}$ Li Fangyu ${ }^{\mathrm{a}, 1}$ \\ ${ }^{a}$ School of Architecture and Design, Southwest Jiaotong University, Chengdu \\ 610000 , Sichuan, China
}

\begin{abstract}
The aim is to explore the innovative design strategies of Lake-age entertainment products in the experience needs of the elderly from the perspective of experience design in the face of the social problem of an ageing population, focusing on the enhancement of the well-being of the elderly population. The SAPAD framework is combined with the design strategy of the aging entertainment experience as the theoretical basis of the study; the in-depth interview method and the semi-structured observation method are used to observe the behaviour of the elderly users, extract the aging behavioural actions and the sub-actions of the interaction with the platform, and analyse the meaning clusters at three levels of experience layer, semantic layer and semantic layer in the meaning dimension of the SAPAD framework, and cluster the meaning clusters and the associated functional components to obtain the design strategy and the design strategy. The clustering analysis is carried out to obtain design strategies and opportunity points. Therefore, the above analysis suggests the possibility of designing and practicing the design of the Lake-Age Entertainment App, which will provide more references for the subsequent research on the field of Lake-Age Entertainment.
\end{abstract}

Keywords. Elderly, SAPAD theory, Active ageing entertainment, Interactive products, Innovative design

\section{Introduction}

China has been an ageing society since 1999 and ageing has become a significant issue for society as a whole ${ }^{[1]}$. Due to the deterioration of the physical functions of the elderly and the inconvenience of mobility and independent living, researchers in China have for a long time focused on the healthcare sector for the elderly, but have lagged behind in the design of ageing experiences. The term "ageing in place" originated in Singapore and other places, using the word "fun" instead of "old" to show the respect and wishes of society for the elderly population ${ }^{[2]}$. Studies published in the Journal of the American Geriatrics Society ${ }^{[3]}$ have shown that the psychological and physical states of older people complement each other, and that older people living alone for long periods of time are prone to depression, anxiety and increased risk of illness or death. Therefore, the design of "ageing experience" can reduce the negative emotions of the elderly and thus reduce the risk of disease by taking care of the psychology of the elderly and providing an open and dynamic communication environment ${ }^{[4]}$, which is a positive response to the "active ageing" and "healthy ageing" proposed by the World Health Organization (WHO). This is in line with the WHO's strategic objectives of "active

${ }^{1}$ Corresponding author at: School of Architecture and Design, Southwest Jiaotong University, 999, Xi An Rd, Chengdu, Sichuan Sheng, China.E-mail address: lfy2009@swjtu.edu.cn (Li.Fangyu). 
ageing" and "healthy ageing" [5]. "Active" corresponds to the emotional attitudes of the general public and older people, and is the core of the study of ageing for fun. Active recreation for older people enhances their sense of fulfilment, belonging and achievement, which greatly affects their subjective well-being ${ }^{[6]}$.

The Semiotic Approach to Product Architecture Design (SAPAD) theoretical framework proposed by Prof. Hu Fei and Prof. Keiichi Sato is an approach to usercentered product design by analyzing the behavior-meaning-object (product) mapping ${ }^{[7]}$. The SAPAD framework consists of three dimensions:

-behaviour dimension (divided into three levels: activity, task and subtask)

-meaning dimension (including the physical, semantic, experiential, semantic, pragmatic and social layers)

-Object dimension (divided into three levels: system, subsystem, and product/portfolio)

The SAPAD framework is combined with the basic framework for the design of ageing recreation experiences, as shown in Figure 1, and is used as the centrepiece for the spiritual support and emotional comfort of the 'third age' population ${ }^{[8]}$. The combined framework can be used to study and analyse the psychological information of the elderly during their ageing behavioural activities, extract the core psychological needs from the psychological information, and obtain the opportunity to design the ageing entertainment app with "fun" instead of "old". The design of the app is based on the "fun" instead of the "old", and the functions and operation experience of the age-appropriate modules are established to enhance the entertainment experience of the elderly in the Internet environment. It can also help to alleviate the problems of monotonous mental life, single activities and emotional depression caused by loneliness ${ }^{[9]}$.

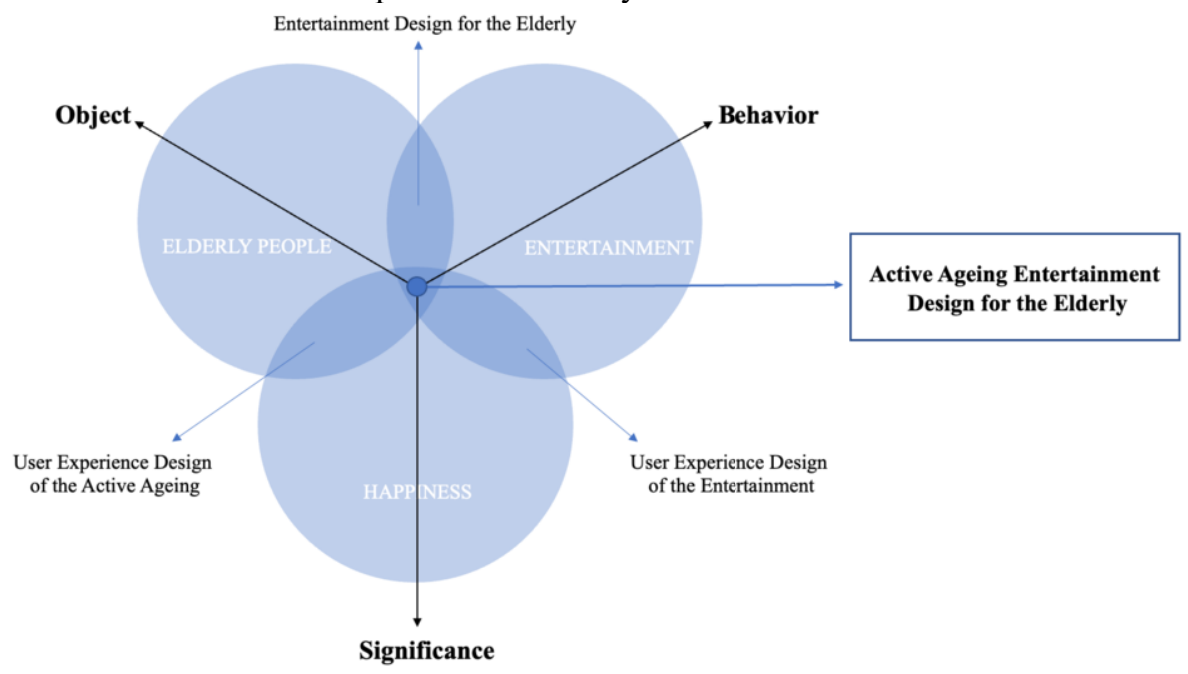

Figure 1. Theoretical framework for the integration of SAPAD and Leaning Recreation Experience Design

\section{An observational analysis of recreational behaviour in an ageing population}

\subsection{Development of the research programme and methodology}

In this study, a healthy retired elderly person aged 60-70 years old was selected to conduct an in-depth interview (one-day follow-up recording) to observe the elderly 
person's autonomous behavioural process in a community setting, and to explore the behavioural activity in which the expression of emotion is "joy". The study will use this behaviour as a theme to investigate the deeper psychological needs of older people. The sample selection criteria and research plan were developed as shown in Figure 2, to understand the behavioural dynamics of older people's recreational activities and the meaning behind the user's behaviour, and to provide material for the SAPAD analysis.

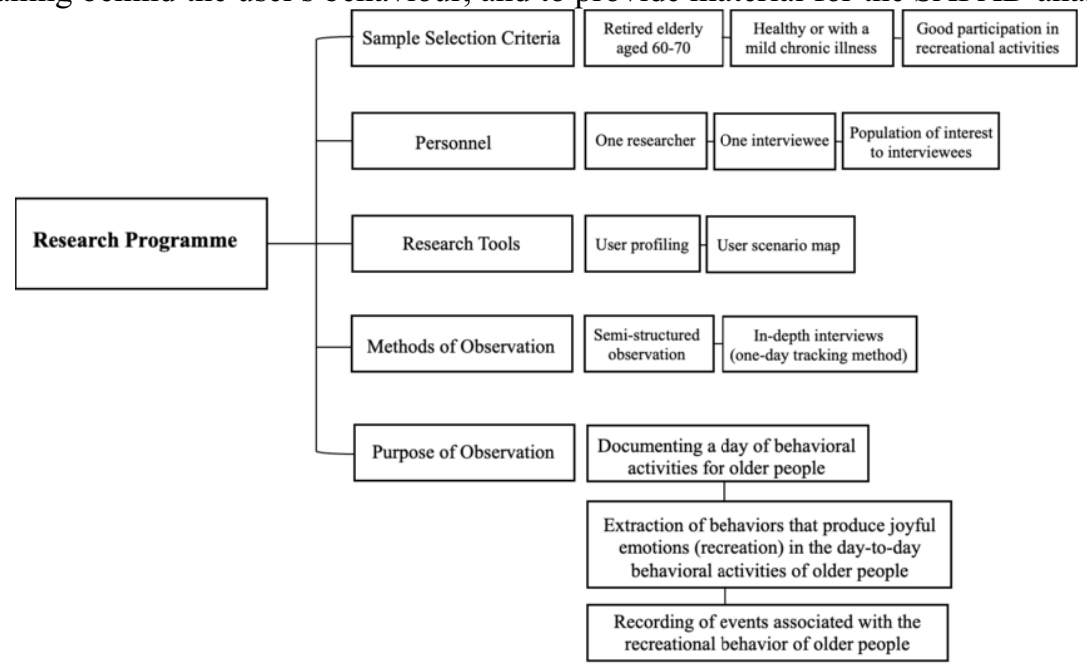

Figure 2. Selection criteria and research programme

\subsection{Behavioural analysis of target users conducting fun-age activities}

Using the selection criteria formulated in 2.1 , the interviewees who fit the study were identified as well as the basic information obtained from them, and a user profile of this interviewer was developed, as shown in Table 1.

Table 1. User profile of interviewees

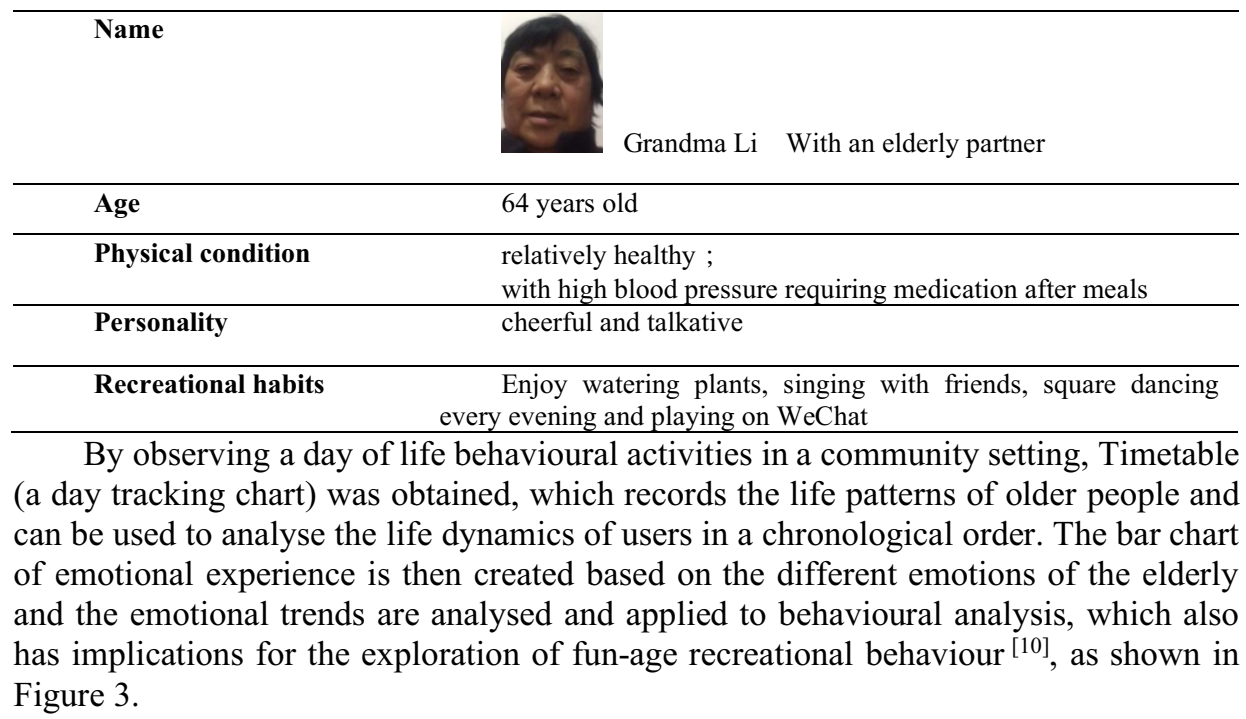




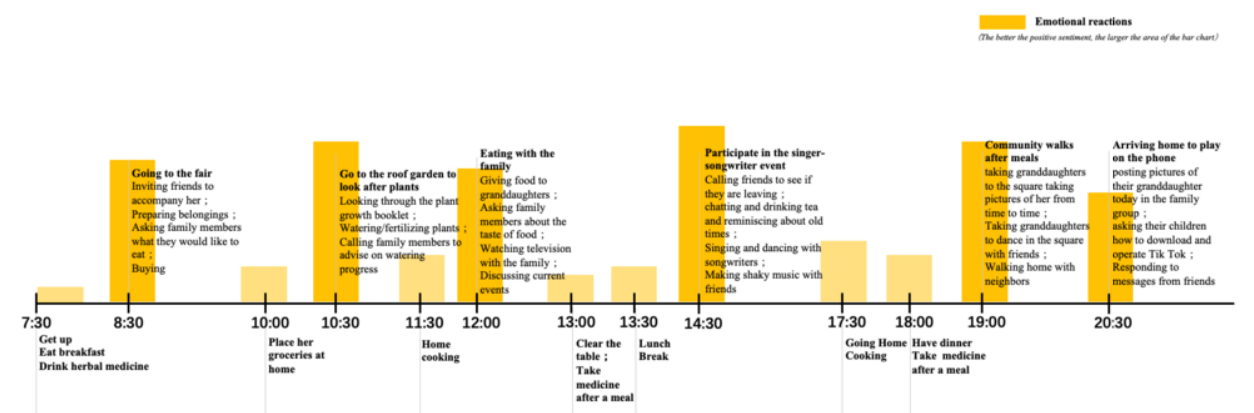

Figure 3. One-day tracking chart of interviewees

Extracting the more emotionally "fun" behaviours from the user's day-to-day behavioural activities and linking these behaviours into a whole range of fun-age recreational activities (Activity of the Active Ageing Entertainment), includingA) Going to the fair;B) Go to the roof garden to look after plants ;C) Eating with the family ;D) Participate in the singer-songwriter event ;E) Community walks after meals ;F) Arriving home to play on the phone. Each part of the activity can involve a corresponding action, as shown in Figure 4. which lists 17 sub-actions in $\mathrm{F}$ action involving platform interactions, as shown in Figure 5.

- F1 Posting photos of granddaughter today in the family group (F1-1 Open the top family group in WeChat; F1-2 Find the key to switch from typing to speech; F1-3 Long press the voice button to chat with family members; F1-4 Video call with son asking how to send photos of granddaughter on WeChat; F1-5 Follow the steps your son or daughter says to successfully share photos)

- F2 Ask children how to download and operate Tik Tok(F2-1 Video call with child about friends filming Tik Tok this afternoon； F2-2 Children teaching the elderly step by step download; F2-3 Children helping to register accounts; F2-4 Very difficult to enter the account password manually; F2-5 Listen to child talk about the basic swiping and tapping actions of Tik Tok; F2-6 Ending a video call)

- F3 Reply to a friend's wechat and try playing Tik Tok(F3-1 Sending messages to friends informing them that she has Shakeology herself ; F3-2 Turn on Tik Tok and try playing it by herself ; F3-3 Often stuck on videos with familiar soundtracks and videos of their friends ; F3-4 Wanted to follow a friend but didn't know how to do it, decided to ask the kids tomorrow ; F3-5 Showing videos to her partner when she found something interesting ; F3-6 Eyes hurt after staring at the video for too long, so she put her phone to her ear and listen to it in a different position) 


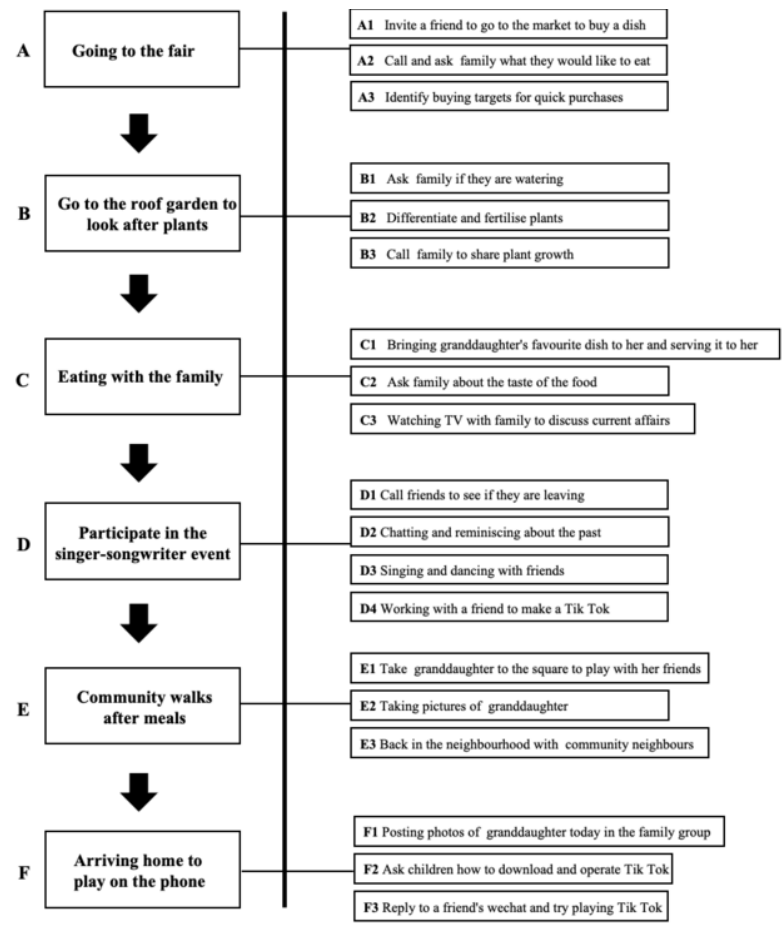

Figure 4. Analysis of recreational behavioural activities in the Lakeview

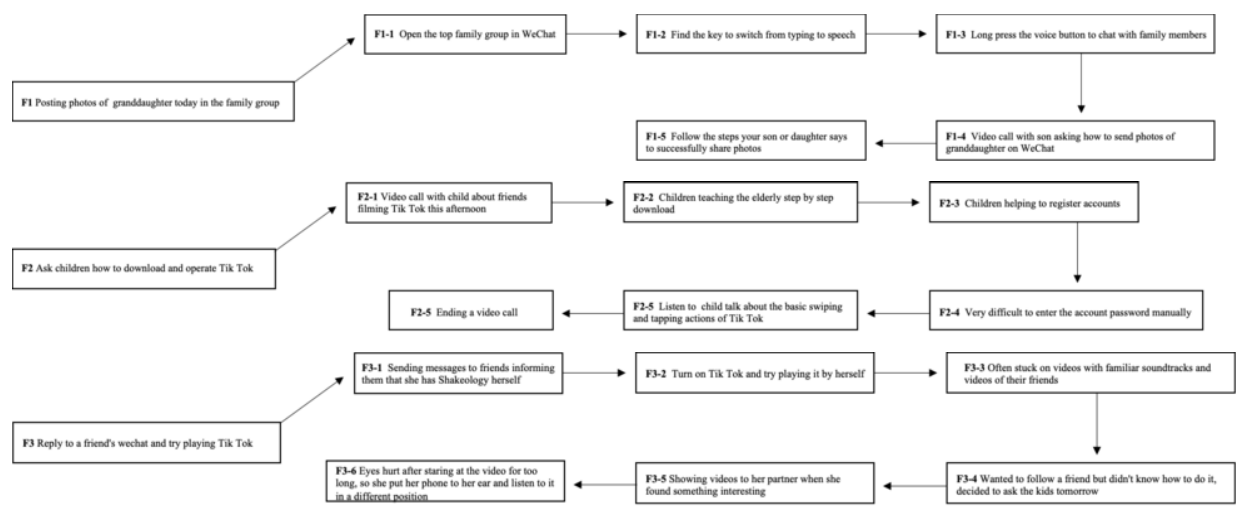

Figure 5. 17 sub-movements in the F-movement

\section{Behaviour-meaning-object analysis based on SAPAD}

\subsection{Core meaning model construction}

Building a meaning model means using the meaning layer in the SAPAD framework to extract the meaning of the corresponding behaviours in six aspects: physical, experiential, semantic, semantic-constructive, pragmatic and social layers ${ }^{[1]}$. First of all, we need to analyse the mapping relationship between the six activities extracted in the first stage 
and the semantic layer in the meaning, to understand the user's emotional experience when performing trivial ageing behaviours (a), and then specifically analyse the mapping relationship between the actions and 17 sub-actions and the meaning when the elderly interact with the mobile phone (b), and combine the two and extract the core meaning cluster, and then complete the meaning construction of ageing entertainment behaviours, as shown in Figure This is shown in Figure 6.

- (a) At the semantic level, cluster analysis revealed six clusters of meaning: (1) active social interaction (2) nostalgia for old things (3) interest in new forms of entertainment (4) physical comfort (5) physical fitness (6) preference for family company.

- (b) At the experiential level, 7 clusters of meaning were identified through cluster analysis: (1) easy to operate (2) good instruction on how to operate (3) avoid eye strain (4) avoid boring home life (5) entertaining in an approachable and simple way (6) gaining satisfaction (7) finding youthfulness; at the semantic level, 6 clusters of meaning were identified through cluster analysis: (1) reminiscing about the past (2) auditory satisfaction (3) Enjoying the sense of multi-participant (4) Not wanting to lag behind the Internet age (5) Good interaction (6) Natural guidance; in the semantic layer, cluster analysis revealed five clusters of meaning: (1) Entertainment needs (2) Sense of achievement (3) Social expression (4) Emotional resonance (5) Self-realisation. The discourse layer focuses on group culture, emphasising the value orientation and ideology of older users.

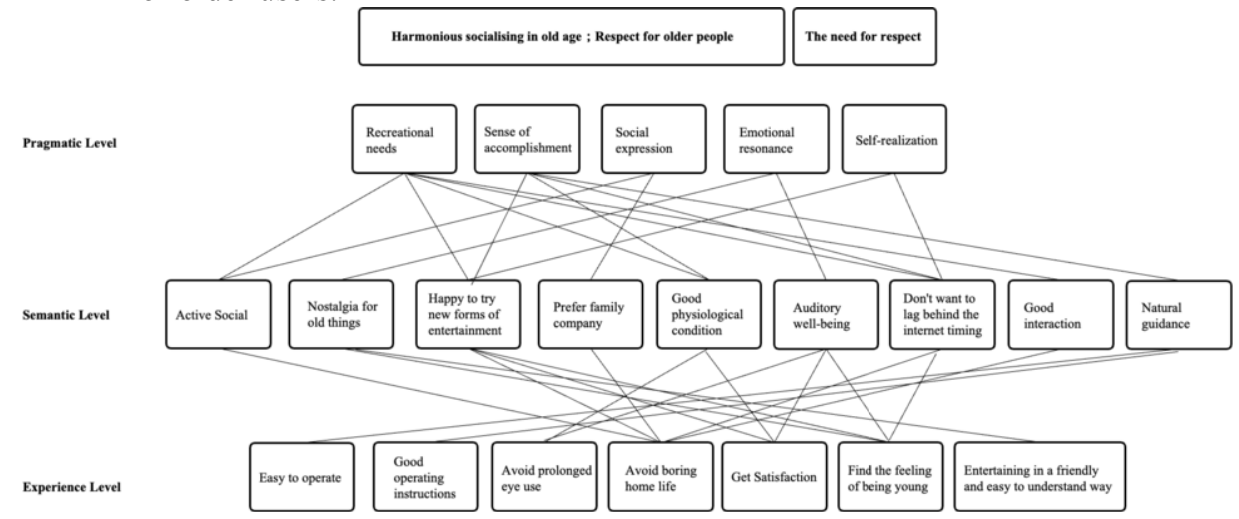

Figure 6. The construction of meaning in the act of recreation at the age of pleasure

\subsection{Design opportunities based on clusters of meaning}

After merging the meaning clusters at the experience and semantic levels, the seven meaning clusters of "easy to use", "natural guidance", "sensory nostalgia", "fun home life", "active social life", "good interaction with recreational activities" and "physical comfort". The clusters were analysed separately. The clustering analysis of the module components related to the seven meaning clusters resulted in the following module components for the use of the Lakeview Entertainment App by the elderly: (1) simple operation: face swipe to register - face swipe to log in - single finger tap gesture - swipe gesture - voice chat - voice message feedback; (2) natural guidance: operation explanation - voice explanation - module colour differentiation; (3) sensory nostalgia: 
tape elements - entertainment records - personal space (4) Interesting home life: entertainment categories - platform amount coins - online mall - singing entertainment chess entertainment; (5) Active social interaction: friend circle - join interest groups chat box - swipe to add friends - entertainment with friends - friend dynamics; (6) Good interaction for entertainment activities: somatosensory mode - sign in with gifts entertainment hours reward; (7) Physical comfort: colour preference settings - sound preference Settings-Somatic interaction.

\section{Meaning-based product design for Leroy recreation app}

Through the study of the meanings generated by the behaviour of elderly users, the functional modules that conform to the user behaviour were derived, and the clustering analysis of the functional components related to the clusters of meanings was combined to derive the user's demand points and explore new age-appropriate entertainment APP products. the information content construction of the APP was based on the determination of the functional components and their meaningful combinations classified by the above analysis, but there was no sound logical information architecture yet, so two elderly people were invited to The combination of APP functional modules using the card classification method, as shown in Figure 7, was used to sort out the preliminary platform information architecture through the elderly users, and then three experts evaluated the preliminary information architecture to sort out the final platform functional modules. The interaction experience of users using the platform is also the focus of attention. Interaction means focusing on the interaction between people and the product, with the user's personal characteristics, operating habits and emotional mobilisation during the operation as the basis of the design, which is also the opportunity point to satisfy the users ${ }^{[12]}$. Therefore, based on the initial functional modules of the platform, a prototype interface was made and two elderly people were invited to conduct usability tests of the interface interaction prototype, as shown in Figure 8.
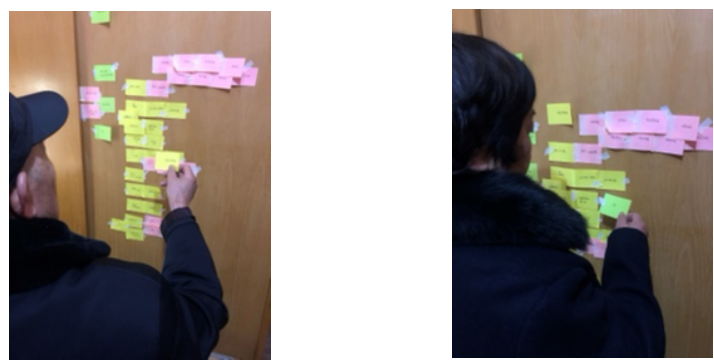

Figure 7. The process of card sorting by the user
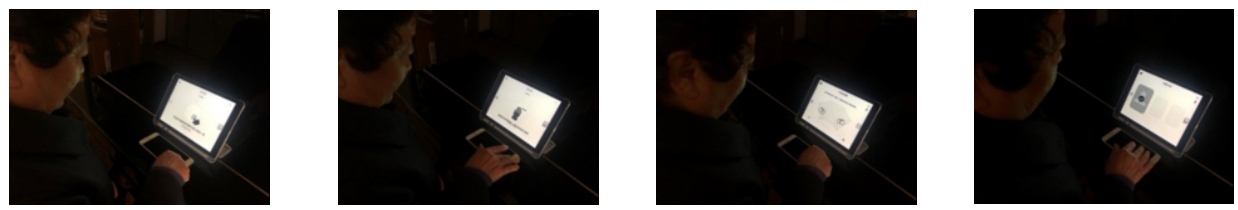

Figure 8. Interface interaction prototype usability testing

The music-based entertainment platform designed for the elderly was derived from several iterations, which effectively improves ease of use and interest persistence by 
catering to user operating habits and culture ${ }^{[13]}$. Older people do not only click on the mobile phone to operate it, they can follow the platform gesture operation instructions to do dynamic movements at the same time, this entertainment behavior is achieved through camera recognition. The simultaneous engagement of senses and behaviour can give older people comprehensive psychological feedback on their interaction with the product [14]. The product is designed to enhance the lives of older people while improving their functional abilities and preventing dementia in later life. The combination of entertainment and fun ageing experience design fully mobilises the participation of the elderly users, bringing them a healthy and leisurely entertainment experience and enhancing their sense of well-being, as shown in Figure 9.
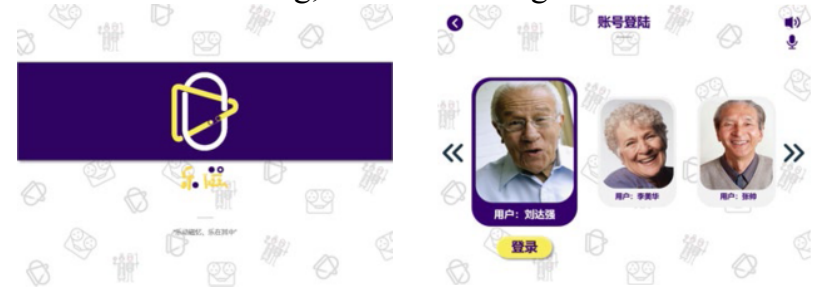

Figure 9(a). Platform cover and login screen
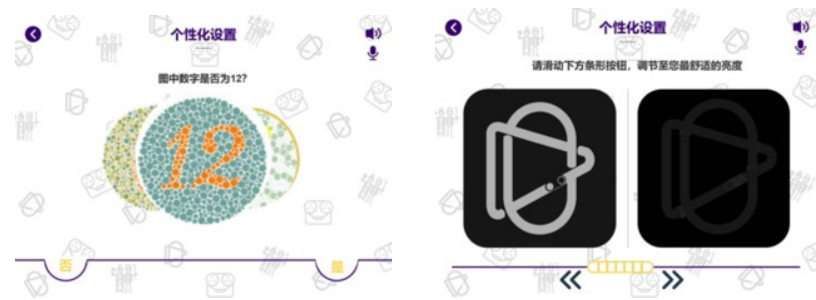

Figure 9(b). Platform colour and brightness adjustment interface
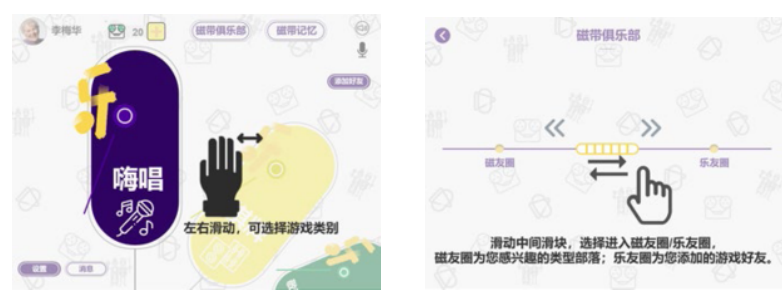

Figure 9(c). Function explanation interface
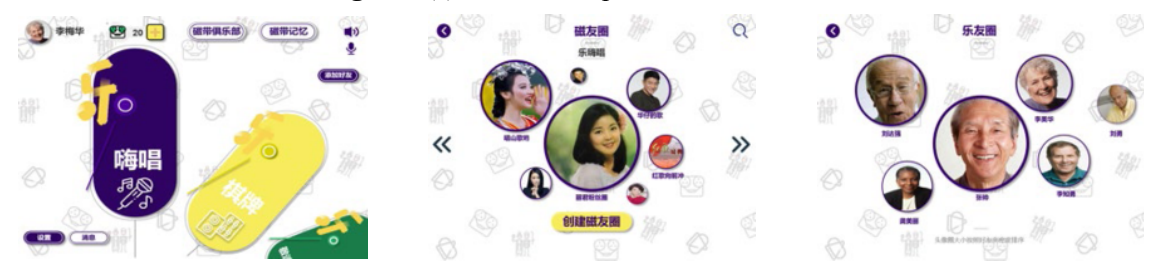

Figure 9(d). Entertainment main interface and interest tribe interface

\section{Discussion and Conclusion}

The importance of the spiritual needs of the ageing population is becoming increasingly important. The government now strongly supports the trend of active ageing and the 
development of smart technology is gradually taking into account the ageing field, which heralds the trend of diversified innovation in the design of ageing innovations. In this paper, based on the theories related to ageing entertainment experience, the SAPAD theoretical framework is applied to explore the three levels of meaning clusters in the daily ageing behaviour of the elderly, and through the meaning clusters, the relevant needs of the elderly and the corresponding module components of the ageing entertainment platform are obtained. Through researching the psychological, physiological and behavioural characteristics of elderly users in their recreational behaviour, we understand what the elderly need and want, in order to determine the innovative elements, functional attributes and technical attributes of the recreational products. However, the study is only based on the general behaviour of individual cases in the SAPAD framework, and more cases will be drawn up for comparison in the future to obtain more valuable implications and generate greater opportunities for innovation in entertainment products for the elderly.

\section{Funding}

Key Project of the Research Center of Aging Business and Industry, Key Research Base of Philosophy and Social Sciences of Sichuan Province (XJLL2019002) ; Key Research Base of Social Sciences of Sichuan Province Sichuan Applied Psychology Research Center, Chengdu Medical College, China (CSXL-212A02) ; General Project of Modern Culture and Design Center, Sichuan Key Research Base of Social Sciences, Sichuan Province (MD21E025)

\section{References}

[1] HU Fei, ZHANG Xi. Designed for the Elderly: the Birth and Evolution of Design Ideas Involving Older People Since 1945[J]. Journal of Nanjing Arts Institute, 2017(6): 39-50+251.

[2] WANG Bing, GU Yu. Singapore's Senior Education: Strategy and Enlightenment[J]. Journal of Hebei Normal University, 2012, 14(2): 19-23.

[3] Yin Xinyu. Research on the design of leaguer games based on emotional experience[D]. Southwest Jiaotong University,2020.

[4] Zhao Chang, Lu Yichen, Zhang Linghao. Research on the design strategy of joyful ageing experience in the context of gamification[J]. Packaging Engineering,2019,40(22):16-23.

[5] Liu W,Jiao P. Research on active aging in international perspective[J]. Journal of Zhongshan University (Social Science Edition),2015,55(1):167-180.

[6] Opinions of Jiangsu Provincial People's Government of the Communist Party of China on Implementing the Decision of the Central Committee of the Communist Party of China and the State Council on Strengthening the Work on Ageing [A]. The turn of the century--a study on the aging of Jiangsu [C]. :Jiangsu Gerontological Society,2001:11.

[7] Hu Fei, Shen Xipeng, Wang Xinyu, Ran Guihong. Research on the overall cabinet design based on SAPAD [J]. Journal of Nanjing Art Institute (Art and Design),2015(05):188-192.

[8] Gao Chao. The caring "third age" design [J]. Decoration, 2012(9):58-65.

[9] LI Fang-yu, Yin Xin-yu, HAN Ting. Application Re- search of Empathy Design in the Design of Senior Clues[J]. Packaging Engineering, 2019, 40(12): 34-41.

[10] Wang Xiaoxia, Wang Ping, Wang Yihua et al. The use of emotional experience curve in the design of humanistic care for elderly products[J]. Science and Technology Perspectives,2019(32):32-32.

[11] Li Tei. Research on the design of educational toys for the elderly based on SAPAD[D]. Guangdong University of Technology, 2020.

[12] Helen Sharp, Yvonne Rogers, Jenny Preece. Interaction Design. Beyond Human-Computer Interaction[M]. John Wiley \& Sons, Inc. 2007. 
[13] Zheng Lixin. Research on the interaction design of age-friendly smart kitchen products[D],2018.

[14] Cao Guozhong, Su Jianhua, Du Xueqing, et al. Research on the design of medical guide robot based on user experience[J]. Art and Design:Theory, 2019, 386(Z1):96-98. 